\title{
Assessment of Extra Cellular Enzymes of Bacteria Isolated from Mangrove Rhizosphere Soil of Different Places of Gujarat in Monsoon Season
}

\author{
A. S. Kharadi*, Megha Patel and F.R. Patel \\ Shri M.M. Patel Institute of Sciences and Research, Kadi Sarva Vishwavidyalaya, Gandhinagar, Gujarat, India; \\ ankitkharadi@gmail.com, meghapatel031@gmail.com, falgunipatel84@gmail.com
}

\begin{abstract}
Background/objectives: Mangroves are one of the most diverse ecosystems on the earth and harbours many organisms, including extremophiles which help the ecosystem to grow and survive despite harsh environment. These microbes produce novel enzymes as well as secondary metabolites that find major applications in extreme environments. Methodology: In this study, total 56 bacteria were isolated from rhizospheric soil of mangrove collected from seven sampling sites of Gujarat, India i.e. Kandla, Mundra, Tuna, Guneri, Machhad, Diu, and Madhwad. These isolates were screened for their production of extracellular hydrolytic enzymes i.e. amylase, protease, cellulase, and catalase. All the isolates were also assessed for their ability to survive in different salt concentration ranging from $0.5 \%$ to $25 \%$ in Zobell marine agar. Findings: After $48 \mathrm{~h}$ of incubation, isolates $\mathrm{K}^{-2}(11) \mathrm{ZO}$ and M10 ${ }^{\circ}(5) \mathrm{NASW}$ had the highest amylase activity, $\mathrm{K} 10^{0}(1) \mathrm{NASW}$ had the highest cellulase activity, and G10-2(9)ZO had the highest protease activity. Among all sites, Madhwad had the highest catalase positives. Three isolates named as $\mathrm{MD} 10^{-2} \mathrm{C} 3, \mathrm{MW} 10^{-3} \mathrm{C} 2$, and MWC4 emerged as possible extreme halotolerant and were able to grow at $25 \%$ of salt concentration while $\operatorname{Diu} 10^{-4} \mathrm{C} 1, \mathrm{MW} 10^{-3}$, and $\mathrm{G} 10^{-1}(5) \mathrm{ZO}$ were also possible halotolerant and were able grow in till $20 \%$ of salt concentration. Application/ improvements: Few isolates that had showed higher extracellular enzyme activity and could be potential high producers that can be used in industries such as detergent industry, textile industries, environmental remediation, etc.
\end{abstract}

Keywords: Mangroves, Enzymes, Cellulase, Amylase, Protease, Extreme Halotolerant

\section{Introduction}

Mangrove forests constitute a large portion of the coastline in the tropical and subtropical regions, where they play an important role in protecting the coastal diversity, protecting the coast from erosion and maintaining water levels. They harbor very rich and diverse living resources that are able to survive in extreme environments. In mangrove sediment, the microorganisms play a crucial role in productivity, conservation, and atmosphere recovery, where they participate within the biogeochemical cycles and provide plant and animals with primary nutritionary sources.,$\underline{2}$ Hence, tropical mangroves are among the foremost productive ecosystems which are being characterised by high rates of organic matter and nutrient cycling. $\underline{3}$ Microbes in this ecosystem convert the dead biological materials from the mangrove plants such as leaves, branches, fruits, and other animal's dead bodies into other simple sources making them available to other living organisms hence helping in running element cycles. These microbes of mangrove ecosystems over millions of years, have evolved morphologically and physiologically to adapt this swampy and saline environments. The enzymes and secondary metabolites produced by these microbes possess the ability to withstand such extreme environments and this characteristic makes them unique.

*Author for correspondence 
Enzymes and metabolites isolated from these microbes are significant in industries, where processes require extreme conditions and use of a chemical process is not allowed or possible.

Gujarat has the longest coastline stretching 1600 $\mathrm{km}$ and has second largest mangrove coverage in India. ${ }^{4}$ This large mangrove ecosystem is a house of many extremophiles such as halophiles, halotolerants, thermophiles, and many other marine microorganisms. Extracellular hydrolytic enzymes such as amylase, protease, and cellulase have potential applications in numerous industries such as food, beverages, bakery products, detergents, textile, environmental remediation, medicinal, clinical, etc. $\stackrel{5-9}{ }$ These enzymes have covered very large portion of the industry and replaced chemicals in many industries. In this study, we screened bacteria for their production of extracellular hydrolytic enzymes such as amylase, protease, cellulase, and catalase isolated from six places of mangrove ecosystems of Gujarat in monsoon season. These isolates were also screened for their ability to survive in different salt concentration.

\section{Materials and Methods}

\subsection{Sample Collection}

Soil samples were collected from six places of mangrove regions of the Gujarat which are situated at the coast line i.e. 1) Kandla, 2) Mundra, 3) Tuna, 4) Guneri, 5) Machhad, 6) Diu, and 7) Madhwad in monsoon season (Figure 1). The sample collection sites had different saline environments i.e. intertidal and estuarine belts. The Machhad site showed the presence of Avicennia officinalis \& Acanthus ilicifolius, whereas Avicennia marina was found to be dominant in remaining 6 sites. The soil samples were collected from the depth of the 10 $\mathrm{cm}$ surrounding the mangrove rhizospheres in triplicates and then stored in sterile plastic bags and kept in ice box till analysed. Physiochemical analysis of soil such as salinity, $\mathrm{pH}$, electrical conductivity, macronutrient (C, N, $\mathrm{P}, \mathrm{K}$, and $\mathrm{S})$, and micronutrient $(\mathrm{Cu}, \mathrm{Fe}, \mathrm{Mn}$, and $\mathrm{Zn})$ was performed for all the soil samples.

\subsection{Isolation of Microbes}

Collected soil samples were enriched in nutrient broth with sea water/Zobell marine broth $\underline{10}$ and incubated at 30 ${ }^{\circ} \mathrm{C}$ at $120 \mathrm{rpm}$ for $72 \mathrm{~h}$. After enrichment, serial dilution of all the samples were made from $10^{0}$ to $10^{-4}$ and were

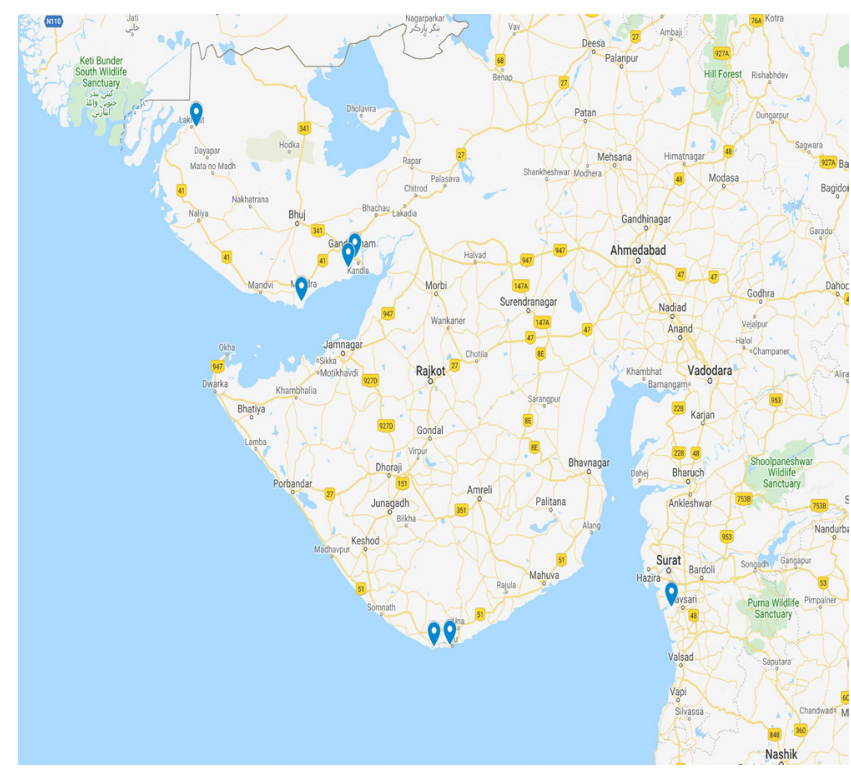

Figure 1. Map of Gujarat, India showing sampling sites.

inoculated by spreading in nutrient agar with sea water/ Zobell marine agar and incubated for $72 \mathrm{~h}$ at $30^{\circ} \mathrm{C}$. At the same time, R2A agar also known as Reasoner's 2A agar11 was used for isolation of slow growing bacteria. For that soil sample were enriched in R2A broth and incubated at $30^{\circ} \mathrm{C}$ at $120 \mathrm{rpm}$ for 10 days and then inoculated by spreading in R2A agar. Morphologically, unique and separate colonies were isolated for further study. The isolated colonies were screened for their extracellular enzyme activity.

\subsection{Primary Screening of Enzymes}

All the isolates were screened for their ability to produce extracellular enzymes i.e. amylase, protease, cellulase, and catalase, and the results were observed after $48 \mathrm{~h}$ of incubation.

\subsubsection{Amylase Screening}

Starch agar with $0.2 \%$ starch as per the technique reported by Hankin and Anagnostakis $\underline{\underline{ }}$ was used for the screening of amylase producers from the isolated bacteria. The amylase positives were identified by a clear zone surrounding the colony on application of iodine solution.

\subsubsection{Protease Screening}

Milk agar with $10 \%$ skimmed milk was used for the screening of protease producers from the isolates. The 
protease producers were identified by a clear zone surrounding the colony.

\subsubsection{Cellulase Screening}

Carboxy methyl cellulose (CMC) agar with $1 \%$ CMC as per the method reported by Method reported by Paterson and Bridge. $\underline{12}$ was used for the screening of cellulase producers. Cellulase positives were identified by a clear zone surrounding the colony on application of iodine solution.

\subsubsection{Catalase Screening}

Catalase activity of the isolates was screened using hydrogen peroxide solution (3\%). The solution of $\mathrm{H}_{2} \mathrm{O}_{2}$ was dropped on bacterial colonies and catalase positives were identified on the basis of effervescence.

\subsubsection{Salt Tolerance Activity}

All the isolates were screened for their ability to survive in a variety of salt concentrations ranging from $0.5 \%$ to $25 \%$.

\section{Results and Discussions}

For this study, the soil samples were collected from different regions of Gujarat, which had different environmental conditions such as Kandla, Mundra, and Tuna are nearby the port areas, Machhad is an estuarine region, Guneri is preserved mangrove area, and Diu and Madhwad have mangrove regions that have human interference. Apart from these environmental conditions, these regions have different mangrove diversity with different salinity levels. The results of the physiochemical analysis of soil collected from these sampling sites were shown in Table 1.

From the soils of above-mentioned sampling sites, total 56 bacteria were isolated which were screened for their enzyme activities as well as their colony characteristics. Enzyme activity on respective agar plates was recorded as the Index of Relative Enzyme Activity and was calculated using the below formula $13, \underline{14}$ :

$$
\text { Index of relative enzyme activity }=\frac{\text { Clear zone diameter }}{\text { Colony diameter }}
$$

The extracellular enzyme activity of each isolate was analysed qualitatively which is represented in Table 2 . Figure 2 represents the total number bacteria isolated from each sampling site and from which how many of them showed particular enzyme activity. From Figure 2, it can be seen that the Mundra, Madhwad, and Guneri had more amylase positives while Tuna and Madhwad had more catalase and protease positive, respectively.

Table 1. Results of physiochemical analysis of the soil

\begin{tabular}{|l|l|l|l|l|l|l|l|}
\hline Sample name & Mundra & Kandla & Tuna & Machhad & Madhwad & Diu & Guneri \\
\hline Longitude & 22.77306 & 23.03316 & 22.97478 & 20.94522 & 20.70635 & 20.71484 & 23.806783 \\
\hline Latitude & 69.70394 & 70.15801 & 70.10283 & 72.85431 & 70.83312 & 70.96765 & 68.808883 \\
\hline $\mathrm{pH}$ & 7.72 & 7.60 & 7.92 & 7.86 & 7.72 & 7.94 & 7.69 \\
\hline Conductivity (S/m) & 13 & 16 & 10 & 6.5 & 10.15 & 11 & 7.40 \\
\hline Salinity (ppt) & 7.5 & 7.3 & 5.6 & 9.5 & 8.7 & 6.2 & 4.1 \\
\hline Organic carbon (\%) & 2.25 & 0.63 & 0.5 & 0.68 & 0.77 & 0.71 & 0.74 \\
\hline Nitrogen (\%) & 0.19395 & 0.054306 & 0.0431 & 0.058616 & 0.066374 & 0.061202 & 0.063788 \\
\hline Phosphorus (ppm) & 6 & 10 & 8 & 10 & 11.9 & 11.2 & 12 \\
\hline Potassium (ppm) & 623.6 & 833.2 & 295.6 & 295.6 & 192.8 & 160 & 123.6 \\
\hline Copper (ppm) & 0.72 & 1.70 & 2.74 & 14.06 & 4.04 & 2.13 & 0.78 \\
\hline Zinc (ppm) & 0.92 & 3.38 & 0.82 & 2.12 & 1.16 & 0.88 & 0.64 \\
\hline Manganese (ppm) & 11.04 & 7.90 & 12.04 & 12.28 & 9.54 & 10.02 & 0.64 \\
\hline Iron (ppm) & 11.80 & 12.06 & 13.04 & 14.10 & 10.52 & 11.34 & 10.66 \\
\hline Sulphur (ppm) & 99.6 & 90.5 & 94.2 & 93.8 & 99.2 & 97.4 & 96.04 \\
\hline Vegetation & $\begin{array}{l}\text { Avicennia } \\
\text { marina }\end{array}$ & $\begin{array}{l}\text { Avicennia } \\
\text { marina }\end{array}$ & $\begin{array}{l}\text { Avicennia } \\
\text { marina }\end{array}$ & $\begin{array}{l}\text { Avicennia officinalis, } \\
\text { Acanthus ilicifolius }\end{array}$ & $\begin{array}{l}\text { Avicennia } \\
\text { marina }\end{array}$ & $\begin{array}{l}\text { Avicennia } \\
\text { marina }\end{array}$ & $\begin{array}{l}\text { Avicennia } \\
\text { marina }\end{array}$ \\
\hline
\end{tabular}


Table 2. Results of enzyme activity of bacteria isolated from their respective sampling site

\begin{tabular}{|c|c|c|c|c|c|}
\hline Sampling site & Name of isolates & Amylase & Cellulase & Protease & Catalase test \\
\hline \multirow{4}{*}{ Diu } & DIU $10^{-3} \mathrm{C} 3$ & 2.8 & 2.8 & 0 & -ve \\
\hline & DIU $10^{-4} \mathrm{C} 3$ & 1.9 & 0 & 3.1 & -ve. \\
\hline & DIU $10^{-4} \mathrm{C} 1$ & 0 & 2.6 & 0 & $-\mathrm{ve}$ \\
\hline & DIU $10^{-1} \mathrm{C} 1$ & 3.3 & 2 & 0 & $+\mathrm{ve}$ \\
\hline \multirow{10}{*}{ Guneri } & $\mathrm{G}^{-1} 0^{-1}(1) \mathrm{ZO}$ & 3 & 0 & 0 & +ve \\
\hline & $\mathrm{G}^{-1} 0^{-1}(3) \mathrm{ZO}$ & 3 & 0 & 2.5 & $+\mathrm{ve}$ \\
\hline & $\mathrm{G}^{-1} 0^{-1}(7) \mathrm{ZO}$ & 4 & 1.7 & 3.3 & -ve \\
\hline & $\mathrm{G} 10^{-1}(2) \mathrm{ZO}$ & 3 & 0 & 2.5 & $+\mathrm{ve}$ \\
\hline & $\mathrm{G} 0^{-1}(5) \mathrm{ZO}$ & 2 & 1.3 & 0 & $+\mathrm{ve}$ \\
\hline & $\mathrm{G}^{2} 0^{-2}(8) \mathrm{ZO}$ & 1.6 & 0 & 0 & -ve \\
\hline & $\mathrm{G} 0^{-2}(9) \mathrm{ZO}$ & 4.5 & 0 & 6.3 & $+\mathrm{ve}$ \\
\hline & G100(8) NASW & 0 & 1.6 & 0 & $+\mathrm{ve}$ \\
\hline & $\mathrm{G10}^{-2}(9) \mathrm{NASW}$ & 0 & 2.3 & 2.3 & -ve \\
\hline & G R2A & 0 & 0 & 3.1 & $-\mathrm{ve}$ \\
\hline \multirow{4}{*}{ Kandla } & $\mathrm{K} 10^{-2}(11) \mathrm{ZO}$ & 5.5 & 0 & 0 & $+\mathrm{ve}$ \\
\hline & $\mathrm{K} 10^{\circ}(1) \mathrm{NASW}$ & 0 & 7 & 2.6 & +ve \\
\hline & $\mathrm{K} 10^{\circ}(4) \mathrm{NASW}$ & 0 & 1.6 & 2.5 & $+\mathrm{ve}$ \\
\hline & K R2A & 2.2 & 1.6 & 2.3 & -ve \\
\hline \multirow{7}{*}{ Machhad } & $\mathrm{MD} 10^{-2} \mathrm{C} 3$ & 3.2 & 3.2 & 1.5 & -ve \\
\hline & $\mathrm{MD} 10^{-3} \mathrm{C} 1$ & 2 & 2.3 & 0 & $-\mathrm{ve}$ \\
\hline & $\mathrm{MD} 10^{-2} \mathrm{C} 1$ & 1.2 & 0 & 2.1 & -ve \\
\hline & $\mathrm{MD} 10^{-3} \mathrm{C} 3$ & 0 & 2.9 & 3.2 & $-\mathrm{ve}$ \\
\hline & $\mathrm{MD} 10^{-2} \mathrm{C} 2$ & 0 & 1.7 & 2.2 & -ve \\
\hline & MD R2A & 1.9 & 0 & 3 & $+\mathrm{ve}$ \\
\hline & MD C4 & 2.2 & 3 & 2.4 & $+\mathrm{ve}$ \\
\hline \multirow{17}{*}{ Madhwad } & MW $10^{-4} \mathrm{C} 1$ & 1.1 & 3.1 & 0 & $+\mathrm{ve}$ \\
\hline & MW $10^{-2} \mathrm{C} 1$ & 1.8 & 0 & 2.2 & -ve \\
\hline & $\mathrm{MW} 10^{-1} \mathrm{C} 2$ & 0 & 3.3 & 2.1 & $-\mathrm{ve}$ \\
\hline & MW $10^{-1} \mathrm{C} 1$ & 2 & 2.2 & 2.1 & $-\mathrm{ve}$ \\
\hline & MW 100 C2 & 3 & 2 & 0 & $+\mathrm{ve}$ \\
\hline & MW $10^{-3} \mathrm{C} 2$ & 0 & 1.1 & 1.1 & -ve \\
\hline & MW $10^{-4} \mathrm{C} 1$ & 2.4 & 0 & 3.1 & $-\mathrm{ve}$ \\
\hline & MW $10^{-4} \mathrm{C} 2$ & .1 .5 & 0 & 1.9 & $-\mathrm{ve}$ \\
\hline & MW $10^{-2} \mathrm{C} 1$ & 0 & 2 & 0 & $+\mathrm{ve}$ \\
\hline & MW C1 & 1.9 & 1.5 & 0 & $+\mathrm{ve}$ \\
\hline & MW C2 & 1.5 & 4 & 0 & -ve \\
\hline & MW C3 & 1.5 & 3.3 & 2 & $+\mathrm{ve}$ \\
\hline & MW C4 & 1.6 & 0 & 2.4 & -ve \\
\hline & MW C5 & 0 & 0 & 2.1 & $+\mathrm{ve}$ \\
\hline & MW $10^{-2}$ & 1.8 & 2.8 & 3.6 & -ve \\
\hline & MW R2A & 1.9 & 1.8 & 0 & $+\mathrm{ve}$ \\
\hline & MW $10^{-3}$ & 1.8 & 2.8 & 3.5 & $+\mathrm{ve}$ \\
\hline
\end{tabular}




\begin{tabular}{|c|c|c|c|c|c|}
\hline Sampling site & Name of isolates & Amylase & Cellulase & Protease & Catalase test \\
\hline \multirow{9}{*}{ Mundra } & 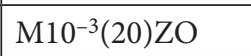 & 2.3 & 0 & 0 & -ve \\
\hline & ${\mathrm{M} 10^{\circ}(9) \mathrm{NASW}}$ & 3.5 & 3 & 0 & -ve \\
\hline & ${\mathrm{M} 10^{\circ}(5) \mathrm{NASW}}$ & 5.5 & 2.2 & 0 & $+\mathrm{ve}$ \\
\hline & ${\mathrm{M} 10^{\circ}(8) \mathrm{NASW}}$ & 2.6 & 2.6 & 0 & -ve \\
\hline & $\mathrm{M10}^{-2}(12) \mathrm{NAS}$ & 0 & 1.4 & 3.2 & -ve \\
\hline & $\mathrm{M} 10^{-2}(2)$ & 0 & 0 & 2.5 & $-\mathrm{ve}$ \\
\hline & $\mathrm{M} 10^{-2}$ & 0 & 0 & 3 & $+\mathrm{ve}$ \\
\hline & M R2A & 1.4 & 0 & 3.8 & $-\mathrm{ve}$ \\
\hline & $\mathrm{M} 10^{-1}$ & 2 & 1.5 & 0 & $-\mathrm{ve}$ \\
\hline \multirow{5}{*}{ Tuna } & $\mathrm{T} 10^{\circ}(16) \mathrm{ZO}$ & 4 & 2.1 & 0 & $+\mathrm{ve}$ \\
\hline & $\mathrm{T} 10^{-1}(13) \mathrm{ZO}$ & 1.3 & 0 & 1.7 & $+\mathrm{ve}$ \\
\hline & $\mathrm{T} 10^{-2}(18) \mathrm{ZO}$ & 3 & 0 & 0 & $+v e$ \\
\hline & $\mathrm{T} 10^{-3}(12) \mathrm{ZO}$ & 1.3 & 2 & 3.3 & $+\mathrm{ve}$ \\
\hline & T R2A & 0 & 1.6 & 2.9 & $+v e$ \\
\hline
\end{tabular}

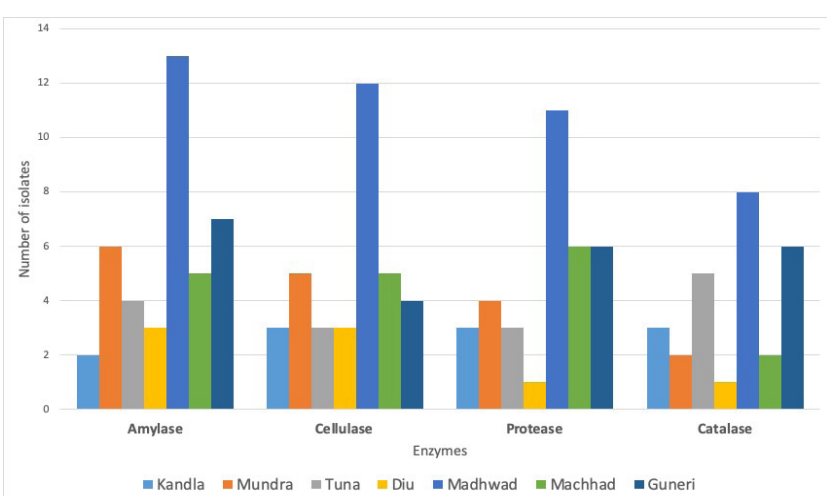

Figure 2. Isolates with their enzyme activity from their respective sampling site.

Kandla had equal number of positives in cellulase, protease, and catalase while Diu had more amylase and cellulase in positives in equal number. Table 3 shows the colony characteristics of these isolates along with results of Gram's reaction.

In Kandla, $\mathrm{K}^{-2} 0^{-2}(11) \mathrm{ZO}$ had highest amylase activity while $\mathrm{K} 10^{\circ}(1) \mathrm{NASW}$ had highest cellulase and protease activity. In Mundra, $\mathrm{M10}^{\circ}(5) \mathrm{NASW}, \mathrm{M10}^{\circ}(9) \mathrm{NASW}$, and MR2A had highest amylase, cellulase, and protease activity, respectively. In Tuna sampling site, $\mathrm{T}^{\circ} 0^{\circ}(16)$ $\mathrm{ZO}$ had the highest amylase and cellulase activity while $\mathrm{T} 10^{-3}(12) \mathrm{ZO}$ had the highest protease activity. In Guneri site, $\mathrm{G} 10^{-2}(9) \mathrm{ZO}$ had highest amylase and protease activity while $\mathrm{G}^{-2} 0^{-2}(9) \mathrm{NASW}$ had highest cellulase activity. In Diu, Diu $10^{-1} \mathrm{C} 1$ had highest amylase activity, while Diu $10^{-3} \mathrm{C} 3$ had highest cellulase activity. In Diu, Diu $10^{-4} \mathrm{C} 3$ is the only protease producer among all. Madhwad had highest number of isolates among all the sites, from which $\mathrm{MW} 10^{\circ} \mathrm{C} 2$ had highest amylase activity, MWC2 had highest cellulase activity, and $\mathrm{MW}^{-2} 0^{-2}$ had highest protease activity. In Machhad, $\mathrm{MD}^{-2} \mathrm{C} 3$ had highest amylase and cellulase activity while $\mathrm{MD}^{-3} 0^{-3} \mathrm{C} 3$ had highest protease activity. For catalase activity, Madhwad showed the highest number of positives i.e. eight isolates followed by Guneri, Tuna, Kandla, Mundra, Machhad, and Diu which showed number of positives six, five, three, two, two, and one isolate(s), respectively.

From all the isolates tested, MDC4, MWC3, MW10-3, and $\mathrm{T}^{-3} 0^{-3}(12) \mathrm{ZO}$ were the isolates showing polyenzyme potential.

Table 4 displays the salt tolerance ability of these isolates which was tested on Zobell marine ager with salt concentration ranging from $0.5 \%$ to $25 \%$. All the bacteria were able to grow on Zobell marine agar with $0.5 \%$ of the salt concentration while majority of the bacteria i.e. $75 \%$ were able to grow on Zobell marine agar with 5\% of salt concentration. The results of the same can be seen in Figure 3. The obtained results showed that 3 isolates ( 1 from Machhad and 2 from Madhwad) i.e. $\mathrm{MD}^{-2} \mathrm{C} 3$, $\mathrm{MW} 10^{-3} \mathrm{C} 2$, and $\mathrm{MWC}$, were able to survive and grow at all salt concentrations provided, starting from $0.5 \%$ to $25 \%$ and emerged as possible extreme halotolerants. Three isolates (each from Diu, Madhwad, and Guneri) i.e. Diu $10^{-4} \mathrm{C} 1, \mathrm{MW} 10^{-3}$, and $\mathrm{G} 10^{-1}(5) \mathrm{ZO}$, had growth 
staring from salt concentration of $0.5 \%$ till $20 \%$ and can be considered as possible extreme halotolerants. The remaining isolates which were able to grow in salt concentration up to $15 \%$ were possible halotolerant. $\frac{15}{5}$

\section{Summary and Conclusion}

In this study, total 56 bacteria were isolated among which total 40 isolates had shown amylase activity, 35 isolates had shown cellulase activity, 34 isolates had shown protease activity, and 27 isolates were catalase positive. Among

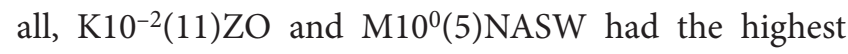
amylase activity which had relative enzyme activity index of 5.5; $\mathrm{K} 10^{\circ}(1) \mathrm{NASW}$ had the highest relative cellulase activity of 7 and $\mathrm{G}^{-2}(9) \mathrm{ZO}$ had the highest relative protease activity of 6.33. In catalase test, Madhwad had the highest catalase positives. Four isolates MDC4, MWC3, $\mathrm{MW}_{10}^{-3}$, and $\mathrm{T}^{-3} 0^{-3}(12) \mathrm{ZO}$ were emerged as polyenzyme producers. Three isolates $\mathrm{MD}^{-2} 0^{-2} \mathrm{C} 3, \mathrm{MW}^{-3} 0^{-3} \mathrm{C} 2$, and MWC4 were able to grow in Zobell marine agar with $25 \%$ salt concentration, whereas $\mathrm{DIU} 10^{-4} \mathrm{C} 1, \mathrm{MW} 10^{-3}$, and $\mathrm{G} 10^{-1}(5) \mathrm{ZO}$ were able to grow in Zobell marine

Table 3. Results of colony characteristics of bacteria isolated from their respective sampling sites

\begin{tabular}{|c|c|c|c|c|c|c|c|c|c|}
\hline $\begin{array}{l}\text { Sampling } \\
\text { site }\end{array}$ & Isolate no. & Size & Shape & Margin & Texture & Elevation & Opacity & Pigmentation & $\begin{array}{l}\text { Gram's } \\
\text { reaction }\end{array}$ \\
\hline \multirow{4}{*}{ Diu } & DIU $10^{-3} \mathrm{C} 3$ & Big & Round & Entire & Smooth & Flat & Opaque & Creamish white & $-\mathrm{ve}$ \\
\hline & DIU $10^{-4} \mathrm{C} 3$ & Small & Round & Entire & Smooth & Flat & Opaque & Creamish white & $+\mathrm{ve}$ \\
\hline & DIU $10^{-4} \mathrm{C} 1$ & Small & Round & Entire & Smooth & Flat & Opaque & Yellow & $+\mathrm{ve}$ \\
\hline & DIU $10^{-1} \mathrm{C} 1$ & Small & Round & Entire & Smooth & Flat & Opaque & Creamish white & $-\mathrm{ve}$ \\
\hline \multirow{10}{*}{ Guneri } & $\mathrm{G}^{-1} 0^{-1}(1) \mathrm{ZO}$ & Big & Round & Entire & Smooth & Flat & Transparent & Creamish white & $-\mathrm{ve}$ \\
\hline & $\mathrm{G} 0^{-1}(3) \mathrm{ZO}$ & Small & Round & Entire & Rough & Flat & Opaque & White & -ve \\
\hline & $\mathrm{G} 0^{-1}(7) \mathrm{ZO}$ & Small & Round & Entire & Smooth & Flat & Opaque & Off white & $-\mathrm{ve}$ \\
\hline & G10-1 (2)ZO & Small & Round & Entire & Smooth & Slightly raised & Opaque & Creamish White & $-\mathrm{ve}$ \\
\hline & $\mathrm{G}^{-1} 0^{-1}(5) \mathrm{ZO}$ & Small & Round & Entire & Smooth & Raised & Opaque & Creamish White & $-\mathrm{ve}$ \\
\hline & $\mathrm{G} 0^{-2}(8) \mathrm{ZO}$ & Small & Round & Entire & Smooth & Flat & Opaque & Creamish white & $-\mathrm{ve}$ \\
\hline & $\mathrm{G} 0^{-2}(9) \mathrm{ZO}$ & Small & Round & Entire & Smooth & Raised & Opaque & Creamish white & $-\mathrm{ve}$ \\
\hline & 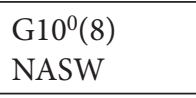 & Small & Round & Entire & Smooth & Flat & Opaque & Creamish white & $-\mathrm{ve}$ \\
\hline & $\begin{array}{l}\mathrm{G} 10^{-2}(9) \\
\text { NAW }\end{array}$ & Small & Round & Entire & Smooth & Flat & Opaque & Brown & + ve \\
\hline & G R2A & Big & Round & Entire & Smooth & Flat & Opaque & Creamish white & $-\mathrm{ve}$ \\
\hline \multirow{4}{*}{ Kandla } & $\mathrm{K} 10^{-2}(11) \mathrm{ZO}$ & Small & Round & Entire & Smooth & Flat & Opaque & White & $-\mathrm{ve}$ \\
\hline & $\begin{array}{l}\mathrm{K} 10^{0}(1) \\
\text { NASW }\end{array}$ & Big & Round & Entire & Smooth & Raised & Opaque & Yellow & - ve \\
\hline & $\begin{array}{l}\mathrm{K} 10^{\circ}(4) \\
\text { NASW }\end{array}$ & Small & Round & Entire & Smooth & Flat & Opaque & Pale yellow & $-\mathrm{ve}$ \\
\hline & K R2A & Small & Round & Entire & Smooth & Raised & Opaque & Creamish white & $+\mathrm{ve}$ \\
\hline \multirow{7}{*}{ Machhad } & $\mathrm{MD} 10^{-2} \mathrm{C} 3$ & Big & Round & Entire & Smooth & Slightly raised & Opaque & Creamish white & $+\mathrm{ve}$ \\
\hline & $\mathrm{MD} 10^{-3} \mathrm{C} 1$ & Small & Round & Entire & Smooth & Flat & Opaque & Creamish yellow & $+\mathrm{ve}$ \\
\hline & $\mathrm{MD} 10^{-2} \mathrm{C} 1$ & Small & Round & Entire & Smooth & Flat & Opaque & Creamish white & $+\mathrm{ve}$ \\
\hline & $\mathrm{MD} 10^{-3} \mathrm{C} 3$ & Small & Round & Entire & Smooth & Flat & Opaque & Creamish white & $+\mathrm{ve}$ \\
\hline & $\mathrm{MD} 10^{-2} \mathrm{C} 2$ & Small & Round & Entire & Smooth & Flat & Opaque & Creamish white & $+\mathrm{ve}$ \\
\hline & MD R2A & Big & Round & Entire & Smooth & Slightly raised & Opaque & Creamish yellow & $-\mathrm{ve}$ \\
\hline & MD C4 & Small & Round & Entire & Smooth & Semi convex & Opaque & Creamish white & $-\mathrm{ve}$ \\
\hline
\end{tabular}




\begin{tabular}{|c|c|c|c|c|c|c|c|c|c|}
\hline $\begin{array}{l}\text { Sampling } \\
\text { site }\end{array}$ & Isolate no. & Size & Shape & Margin & Texture & Elevation & Opacity & Pigmentation & $\begin{array}{l}\text { Gram's } \\
\text { reaction }\end{array}$ \\
\hline \multirow{17}{*}{ Madhwad } & $\mathrm{MW} 10^{-4} \mathrm{C} 1$ & Big & Round & Entire & Smooth & Slightly raised & Opaque & Creamish yellow & +ve \\
\hline & $\mathrm{MW} 10^{-2} \mathrm{C} 1$ & Small & Round & Entire & Smooth & Flat & Opaque & Creamish white & $-\mathrm{ve}$ \\
\hline & $\mathrm{MW} 10^{-1} \mathrm{C} 2$ & Small & Round & Entire & Smooth & Slightly raised & Opaque & Creamish White & + ve \\
\hline & $\mathrm{MW} 10^{-1} \mathrm{C} 1$ & Small & Round & Entire & Smooth & Flat & Opaque & Yellow & +ve \\
\hline & MW 100 C2 & Big & Round & Uneven & Rough & Flat & Opaque & Creamish white & $-\mathrm{ve}$ \\
\hline & $\mathrm{MW} 10^{-3} \mathrm{C} 2$ & Small & Round & Entire & Smooth & Flat & Opaque & Creamish white & $+v e$ \\
\hline & MW $10^{-4} \mathrm{C} 1$ & Big & Round & Entire & Smooth & Flat & Opaque & Yellow & + ve \\
\hline & MW $10^{-4} \mathrm{C} 2$ & Small & Round & Entire & Smooth & Flat & Opaque & Creamish white & $+\mathrm{ve}$ \\
\hline & MW $10^{-2} \mathrm{C} 1$ & Big & Round & Entire & $\begin{array}{l}\text { Dew } \\
\text { Drop }\end{array}$ & Convex & Opaque & Creamish yellow & $+\mathrm{ve}$ \\
\hline & MW C1 & Small & Round & Entire & Smooth & Slightly raised & Opaque & Creamish white & $-\mathrm{ve}$ \\
\hline & MW C2 & Small & Round & Entire & Smooth & Flat & Opaque & Yellow & $+\mathrm{ve}$ \\
\hline & MW C3 & Big & Round & Uneven & Rough & Flat & Opaque & Creamish white & $-\mathrm{ve}$ \\
\hline & MW C4 & Small & Round & Entire & Smooth & Flat & Opaque & Creamish white & $+\mathrm{ve}$ \\
\hline & MW C5 & Big & Round & Entire & Smooth & Slightly raised & Opaque & Yellow & -ve \\
\hline & MW $10^{-2}$ & Small & Round & Entire & Smooth & Flat & Opaque & Creamish white & $-\mathrm{ve}$ \\
\hline & MW R2A & Big & Round & Entire & Rough & Convex & Opaque & Yellow & $+\mathrm{ve}$ \\
\hline & MW $10^{-3}$ & Big & Round & Entire & Rough & Flat & Opaque & Creamish white & $-\mathrm{ve}$ \\
\hline \multirow{9}{*}{ Mundra } & $\begin{array}{l}{\mathrm{M} 10^{-3}(20)}_{\mathrm{ZO}}\end{array}$ & Small & Round & Entire & Rough & Raised & Opaque & Creamish white & $-\mathrm{ve}$ \\
\hline & $\begin{array}{l}\mathrm{M} 10^{\circ}(9) \\
\text { NASW }\end{array}$ & Small & Round & Entire & Rough & Raised & Opaque & Off white & $+\mathrm{ve}$ \\
\hline & $\begin{array}{l}{\mathrm{M} 10^{0}(5)}^{\text {NASW }}\end{array}$ & Small & Round & Entire & Smooth & Flat & Opaque & Yellow & $-\mathrm{ve}$ \\
\hline & $\begin{array}{l}\mathrm{M} 10^{\circ}(8) \\
\text { NASW }\end{array}$ & Small & Round & Entire & Rough & Flat & Opaque & Creamish white & $-\mathrm{ve}$ \\
\hline & $\begin{array}{l}\mathrm{M} 10^{-2}(12) \\
\text { NAS }\end{array}$ & Small & Round & Entire & Smooth & Flat & Opaque & Creamish white & $-\mathrm{ve}$ \\
\hline & $\mathrm{M} 10^{-2}(2)$ & Small & Round & Entire & Rough & Raised & Opaque & Creamish white & $-\mathrm{ve}$ \\
\hline & $\mathrm{M} 10^{-2}$ & Small & Round & Entire & Rough & Raised & Opaque & Creamish white & -ve \\
\hline & M R2A & Small & Round & Entire & Smooth & Raised & Opaque & Off white & $-\mathrm{ve}$ \\
\hline & $\mathrm{M} 10^{-1}$ & Small & Round & Entire & Smooth & Raised & Opaque & Creamish white & $-\mathrm{ve}$ \\
\hline \multirow{5}{*}{ Tuna } & $\mathrm{T} 10^{0}(16) \mathrm{ZO}$ & Small & Round & Entire & Smooth & Flat & Opaque & Creamish white & - ve \\
\hline & $\mathrm{T}^{1} 0^{-1}(13) \mathrm{ZO}$ & Small & Round & Entire & Rough & Flat & Opaque & Creamish white & $-\mathrm{ve}$ \\
\hline & $\mathrm{T}^{10^{-2}}(18) \mathrm{ZO}$ & Big & Round & Entire & Smooth & Flat & Opaque & Off white & +ve \\
\hline & 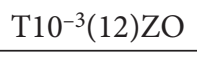 & Small & Round & Entire & Rough & Raised & Opaque & Creamish white & $-\mathrm{ve}$ \\
\hline & T R2A & Small & Round & Entire & Smooth & Flat & Opaque & Creamish white & $+\mathrm{ve}$ \\
\hline
\end{tabular}

agar with $20 \%$ salt concentration and hence emerged as possible extreme halophiles. Higher salinity levels at the sampling sites Madhwad and Machhad may lead to higher number of extreme halotolerants from these sites. From the application point of view, this study provides useful 
Table 4. Salt tolerance ability of the isolated bacteria from their respective sampling site

\begin{tabular}{|c|c|c|c|c|c|c|c|}
\hline $\begin{array}{l}\text { Sampling } \\
\text { site }\end{array}$ & $\begin{array}{l}\text { Name of } \\
\text { isolates }\end{array}$ & $0.5 \%$ & $5 \%$ & $10 \%$ & $15 \%$ & $20 \%$ & $25 \%$ \\
\hline \multirow{4}{*}{ Diu } & DIU $10^{-3} \mathrm{C} 3$ & + & & & & & \\
\hline & DIU $10^{-4} \mathrm{C} 3$ & + & + & + & & & \\
\hline & DIU $10^{-4} \mathrm{C} 1$ & + & + & + & + & + & \\
\hline & DIU $10^{-1} \mathrm{C} 1$ & + & + & + & + & & \\
\hline \multirow{10}{*}{ Guneri } & $\mathrm{G}^{-1}(1) \mathrm{ZO}$ & + & + & + & + & & \\
\hline & $\mathrm{G} 10^{-1}(3) \mathrm{ZO}$ & + & + & + & + & & \\
\hline & $\mathrm{G}^{-1} 0^{-1}(7) \mathrm{ZO}$ & + & + & & & & \\
\hline & $\mathrm{G} 0^{-1}(2) \mathrm{ZO}$ & + & + & & & & \\
\hline & $\mathrm{G}_{10}^{-1}(5) \mathrm{ZO}$ & + & + & + & + & + & \\
\hline & $\mathrm{G} 0^{-2}(8) \mathrm{ZO}$ & + & + & & & & \\
\hline & $\mathrm{G} 0^{-2}(9) \mathrm{ZO}$ & + & + & & & & \\
\hline & 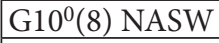 & + & + & & & & \\
\hline & ${\mathrm{G} 10^{-2}(9) \mathrm{NAW}}$ & + & + & + & + & & \\
\hline & G R2A & + & + & & & & \\
\hline \multirow{4}{*}{ Kandla } & $\mathrm{K} 10^{-2}(11) \mathrm{ZO}$ & + & + & & & & \\
\hline & K10(1) NASW & + & + & + & & & \\
\hline & $\mathrm{K} 10^{\circ}(4) \mathrm{NASW}$ & + & + & + & & & \\
\hline & K R2A & + & + & + & & & \\
\hline \multirow{7}{*}{ Machhad } & $\mathrm{MD} 10^{-2} \mathrm{C} 3$ & + & + & + & + & + & + \\
\hline & $\mathrm{MD} 10^{-3} \mathrm{C} 1$ & + & + & + & & & \\
\hline & $\mathrm{MD} 10^{-2} \mathrm{C} 1$ & + & + & & & & \\
\hline & $\mathrm{MD} 10^{-3} \mathrm{C} 3$ & + & & & & & \\
\hline & MD $10^{-2} \mathrm{C} 2$ & + & & & & & \\
\hline & MD R2A & + & & & & & \\
\hline & MD C4 & + & + & + & + & & \\
\hline \multirow{17}{*}{ Madhwad } & MW $10^{-4} \mathrm{C} 1$ & + & + & & & & \\
\hline & $\mathrm{MW} 10^{-2} \mathrm{C} 1$ & + & + & + & + & & \\
\hline & MW $10^{-1} \mathrm{C} 2$ & + & & & & & \\
\hline & $\mathrm{MW} 10^{-1} \mathrm{C} 1$ & + & & & & & \\
\hline & MW 100 C2 & + & + & + & & & \\
\hline & $\mathrm{MW} 10^{-3} \mathrm{C} 2$ & + & + & + & + & + & + \\
\hline & MW $10^{-4} \mathrm{C} 1$ & + & + & & & & \\
\hline & $\mathrm{MW} 10^{-4} \mathrm{C} 2$ & + & & & & & \\
\hline & $\mathrm{MW} 10^{-2} \mathrm{Cl}$ & + & & & & & \\
\hline & MW C1 & + & + & + & & & \\
\hline & MW C2 & + & + & & & & \\
\hline & MW C3 & + & & & & & \\
\hline & MW C4 & + & + & + & + & + & + \\
\hline & MW C5 & + & & & & & \\
\hline & MW $10^{-2}$ & + & + & + & & & \\
\hline & MW R2A & + & & & & & \\
\hline & MW $10^{-3}$ & + & + & + & + & + & \\
\hline \multirow{9}{*}{ Mundra } & $\mathrm{M} 10^{-3}(20) \mathrm{ZO}$ & + & + & + & + & & \\
\hline & $\mathrm{M10}^{\circ}(9) \mathrm{NASW}$ & + & & & & & \\
\hline & $\mathrm{M10}^{\circ}(5) \mathrm{NASW}$ & + & + & + & + & & \\
\hline & $\mathrm{M} 10^{\circ}(8) \mathrm{NASW}$ & + & + & & & & \\
\hline & $\mathrm{M} 10^{-2}(12) \mathrm{NAS}$ & + & + & + & + & & \\
\hline & $\mathrm{M} 10^{-2}(2)$ & + & & & & & \\
\hline & $\mathrm{M} 10^{-2}$ & + & & & & & \\
\hline & M R2A & + & + & & & & \\
\hline & M 10-1 & + & + & & & & \\
\hline
\end{tabular}

\begin{tabular}{|l|l|l|l|l|l|l|l|}
\hline \multirow{5}{*}{ Tuna } & T10 $10^{0}(16) \mathrm{ZO}$ & + & + & + & + & & \\
\cline { 2 - 9 } & $\mathrm{T} 10^{-1}(13) \mathrm{ZO}$ & + & + & + & & & \\
\cline { 2 - 9 } & $\mathrm{T} 10^{-2}(18) \mathrm{ZO}$ & + & + & + & + & & \\
\cline { 2 - 9 } & $\mathrm{T} 10^{-3}(12) \mathrm{ZO}$ & + & + & & & & \\
\cline { 2 - 9 } & $\mathrm{T} 2 \mathrm{~A}$ & + & + & & & & \\
\hline
\end{tabular}

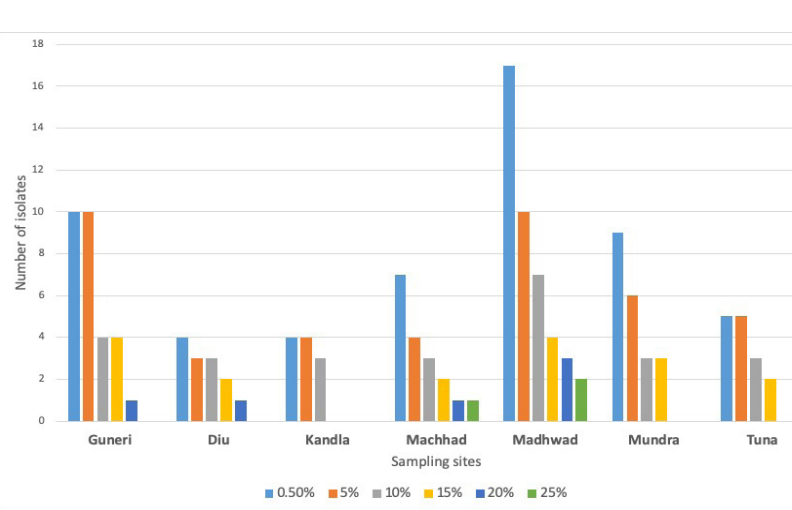

Figure 3. Representation of salt tolerance ability of the isolated bacteria.

information about the bacteria prevailing in mangrove ecosystem of Gujarat.

\section{References}

1. Andreote FD, Jiménez DJ, Chaves D, Dias ACF, Luvizotto DM, Dini-Andreote. The Microbiome of Brazilian Mangrove Sediments as Revealed by Metagenomics. PLoS ONE. 2012, 7 (6), pp. 1-14.

2. Holguin G, Vazquez P, Bashan Y. The role of sediment microorganisms in the productivity, conservation, and rehabilitation of mangrove ecosystems: an overview. Biology and fertility of soils. 2001, 33 (4), pp. 265-278.

3. Nedwell D, Blackburn T, Wiebe W. Dynamic nature of the turnover of organic carbon, nitrogen and sulphur in the sediments of a Jamaican mangrove forest. Marine Ecology Progress Series. 1994, pp. 223-231.

4. Comeau AM, Li WKW, Tremblay JÉ, Carmack EC, Lovejoy C. Arctic Ocean Microbial Community Structure before and after the 2007 Record Sea Ice Minimum. PLOS ONE. 2011, 6 (11), pp. 1-12.

5. Gupta R, Gigras P, Mohapatra H, Goswami VK, Chauhan B. Microbial $\alpha$-amylases: a biotechnological perspective. Process biochemistry. 2003, 38 (11), pp. 1599-616.

6. Kandra L. a-Amylases of medical and industrial importance. Journal of Molecular Structure: THEOCHEM. 2003, 666, pp. 487-498.

7. Pandey A, Nigam P, Soccol CR, Soccol VT, Singh D, Mohan R. Advances in microbial amylases. Biotechnology and applied biochemistry. 2000, 31, pp. 135-152. 
8. Hankin L, Anagnostakis SL. The Use of Solid Media for Detection of Enzyme Production by Fungi. Mycologia. 1975, pp. 67, pp. 597.

9. Jadeja S, Patel N, Kapadia C. Microflora of Soil in the Tidal Zone of Mangrove Ecosystem. Trends in Biosciences. 2017, 10 (48), pp. 9539-9545.

10. ZoBell CE. Studies on marine bacteria. I. The cultural requirements of heterotrophic aerobes. Journal of Marine Research. 1941, 4, pp. 42-75.

11. Reasoner DJ and Geldreich EE. A new medium for the enumeration and subculture of bacteria from potable water. Applied and Environmental Microbiology. 1985, 49 (1), pp. $1-7$.
12. Paterson RRM, Bridge PD. Biochemical techniques for filamentous fungi. Wallingford: CAB INTERNATIONAL; 1994. $\mathrm{x}+125 \mathrm{pp}$

13. Teather RM, Wood PJ. Use of Congo red-polysaccharide interactions in enumeration and characterization of cellulolytic bacteria from the bovine rumen. Applied and Environmental Microbiology. 1982, 43 (4), pp. 777-780.

14. Bradner J, Gillings M, Nevalainen K. Qualitative assessment of hydrolytic activities in Antarctic microfungi grown at different temperatures on solid media. World Journal of Microbiology and Biotechnology. 1999, 15 (1), pp. 131-132.

15. Margesin R, Schinner F. Potential of halotolerant and halophilic microorganisms for biotechnology. Extremophiles. 2001;5(2), pp. 73-83. 